\title{
Optimization of gas turbine engine control using dynamic programming
}

\author{
Venedikt Kuz'michev, Ilia Krupenich, Evgeny Filinov and Andrey Tkachenko \\ Samara National Research University, Department of Aircraft Engine Theory, Samara, Russian Federation
}

\begin{abstract}
The aim of engine control optimization is to derive the optimal control law for engine operation managing during the aircraft flight. For numerical modeling a continuous flight process defined by a system of differential equations is replaced by a discrete multi-step process. Values of engine control parameters in particular step uniquely identify a system transitions from one state to another. The algorithm is based on the numerical method of dynamic programming and the Bellman optimality principle. The task is represented as a sequence of nested optimization subtasks, so that control optimization at the first step is external to all others. The optimum control function can be determined using the minimax principle of optimality. Aircraft performance calculation is performed by numerical integration of differential equations of aircraft movement.
\end{abstract}

\section{Introduction}

The constantly increasing prices of jet fuels stimulate continuous research and development efforts in improving the fuel efficiency of commercial aviation. Last decades have shown that fuel efficiency improvements have slowed down notably, bringing complex versatile approaches to reducing the life-cycle cost of aviation. These approaches include developing new aircraft technologies and designs, profound airframe-propulsion integration, use of alternative fuels, advanced thermodynamic cycles including variable cycle, optimization of propulsion control with an account to transient modes of operation, including onboard control systems [1-7].

Recently, propulsion control optimization issues have been addressed by a substantial number of research groups [8-18]. The article addresses the development of propulsion control optimization method on the basis of operation envelope of aircraft with an account for transient modes of operation of engines and aircraft. The described task is a part of complex efforts of Samara University in developing the optimal design methodology of aircraft gas turbine engines, covering all stages and aspects of life cycle.

With the external conditions and the operating mode varying during the flight of an aircraft, the parameters of the gas turbine engine (GTE) working process change in accordance with the engine control program and the general laws of joint operation of its elements. The engine control program substantially influences the aircraft behavior during the flight, flight time, required amount of fuel per flight, and eventually the aircraft efficiency criteria [19].
Numerical integration of the differential equation system of aircraft motion may be used to calculate aircraft performance criteria at the conceptual design stage of a gas turbine engine. In this case, the parameters characterizing the efficiency of the aircraft are functionals, and their values depend on the engine control program.

Optimization of GTE control program using the aircraft motion model is not solvable by traditional analytical methods of the calculus of variations. Therefore, the solution may be obtained using numerical methods of dynamic programming.

The continuous process described by the system of differential equations is substituted by a discrete multi-step process with each stage corresponding to certain values of the phase coordinates (Fig. 1).

The method of dynamic programming is based on Bellman's optimality principle [20, 14], formulated for solving a wide range of control problems that break up into a series of successive steps. The control function is described as a set of control steps, each characterized by the values of control parameters.

For a given initial state of the system, the sequence of control steps uniquely determines the sequence of system transitions from initial state to final.

\section{GTE control optimizing}

The task of GTE control optimizing is to find such a sequence of step controls which provides the optimal value of the objective function $[21,22]$.

Control function on the $\mathrm{i}$-th step called a control step and is denoted by $u^{(i)}$. If the number of stages is $\mathrm{n}$, then the optimization task is to find the sequence of control steps, i.e. the set $u=\left(u^{(1)}, u^{(2)}, \ldots u^{(n)}\right)$. Since the initial state of the system is specified, the sequence of control steps uniquely 
determines the sequence of system transitions from one state to another.

Mathematically the task may be described as follows:

$$
Y(u)=\Psi(F(u)) \rightarrow o p t,
$$

where $\mathrm{Y}(\mathrm{u})$ - goal function; $\mathrm{F}(\mathrm{u})$ - control function.

The state parameters of the system at the end of process defines each of efficiency criterion:

$$
S^{(n)}(u)=\left(S_{1}^{(n)}(u), S_{2}^{(n)}(u), \ldots S_{l}^{(n)}(u)\right),
$$

where $S^{(n)}(u)$ - set of state parameters (state of the system) at the last (n-th) step, thus defining the goal function itself:

$$
Y(u)=\Psi^{\prime}\left(S^{(n)}(u)\right)
$$

The state of the system at the i-th step $S^{(i)}$, depends on the previous state $S^{(i-1)}$ and the control function at this step $u^{(i)}$ :

$$
S^{(i)}=f\left(S^{(i-1)}, u^{(i)}\right)
$$

According to the Bellman principle, the optimum objective function for the current system state $S^{(i)}$ is a prerequisite for control optimization at later stages.

Thus, for the last $\mathrm{n}$ - th step the optimal transition to the final state (provided by optimal control step $u^{(n)}$ may easily be found using the numerical methods of parametric optimization:

$$
\bar{Y}(u)=\underset{u^{(n)}}{\operatorname{opt}} \Psi^{\prime}\left(S^{(n-1)}(u)\right) .
$$

Optimization at the penultimate step (n-1) is actually the same operation, but for each variant of control step $u^{(n-}$ 1) additional nested optimization of $u^{(n)}$ is performed. Optimization of the previous steps includes the recurrent optimization of control at all the following steps as an optimal control of each step (flight path section) can be carried out only after optimization at all subsequent stages, and determination of the objective function [23].

Thus, the task of finding the optimal process control function consisting of $n$ steps can be reduced to $n$ nested parametric optimization problems. Thus the problem of determining the optimal control at the $n$-th step is embedded in the task of determining optimal control at the (n-1)-th step, and so on, and the problem of determining the optimal control at the first step is external to all the others[24].

Based on the described method, the following algorithm of solving the problem of optimizing the control of the gas turbine engine may be defined:

1. The flight is considered as a discrete process, that is, the entire flight path is divided into small sections, and the change in the state parameters of the aircraft is described by the values at the boundaries of these sections.

2. The initial values of the control parameters in each section of the trajectory and the values of the state parameters of the aircraft at the starting point are set. Optimization begins with the calculation of the first section of the trajectory.

3. State parameters at the end of each step are calculated as a function of the state parameters at the beginning and the current value of control step value.

4. Then, the parameters of a successive section of the flight path are calculated in the same manner.

5. As the parameters of all the sections are calculated, the values of the efficiency criteria of the aircraft and the objective function are calculated.

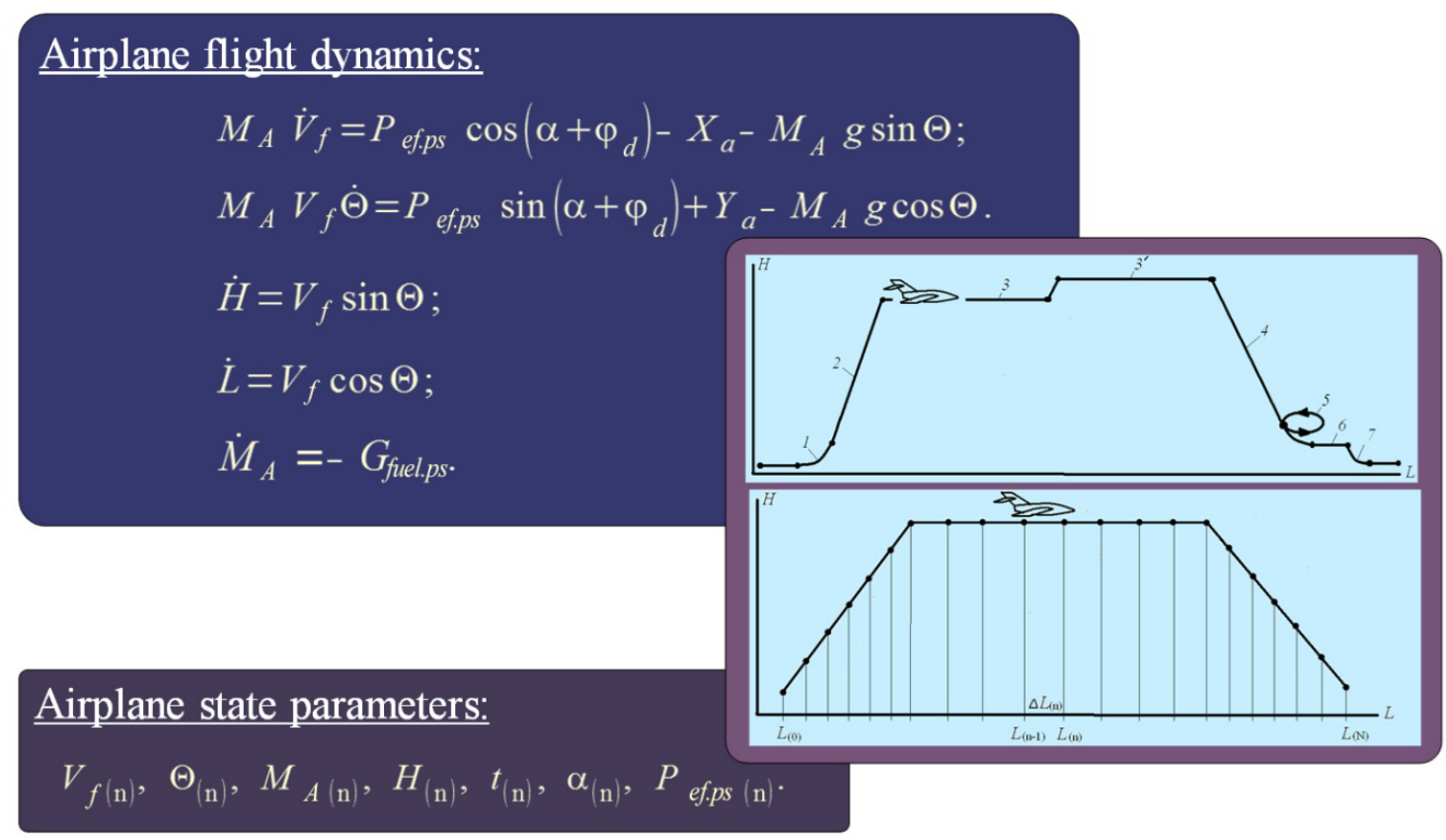

Figure 1. Aircraft flight process modelling. 
6. The convergence condition of the solution is checked for the current values of the gas turbine engine control parameters at this section of the trajectory. If the convergence condition is not satisfied, new values of the control parameters are determined and the calculation is repeated from the step 3 . If the convergence condition of the solution is satisfied and the current path segment is the first, the calculation is finished; otherwise, the previous segment of the trajectory is optimized (beginning from the step 4).

Block diagram of the algorithm for optimizing control of a three-step process as an example is shown in Fig. 2.

Each of the nested GTE control optimization tasks on a separate trajectory section can be solved using one of the widely used numerical parametric optimization methods.
Different criteria for assessing the effectiveness of aircraft correspond to various options of controlling its engines $[25,26]$.

Therefore, the method of calculating the objective function is an important issue in developing a method for optimizing the control of gas turbine engines. In order to determine the compromise version of the GTE control that meets the set of aircraft efficiency criteria, it is necessary to use some optimality principle.

The minimax optimality principle is suitable for most of the technical problems. According to this principle, the minimized value is the maximum value of the set of normalized efficiency criteria:

$$
Y(u)=\max _{j}\left(\rho_{j} F_{j}^{\text {norm }}(u)\right) \rightarrow \min ,
$$

where $\rho$ - significance factor of $\mathrm{j}$-th criterion;

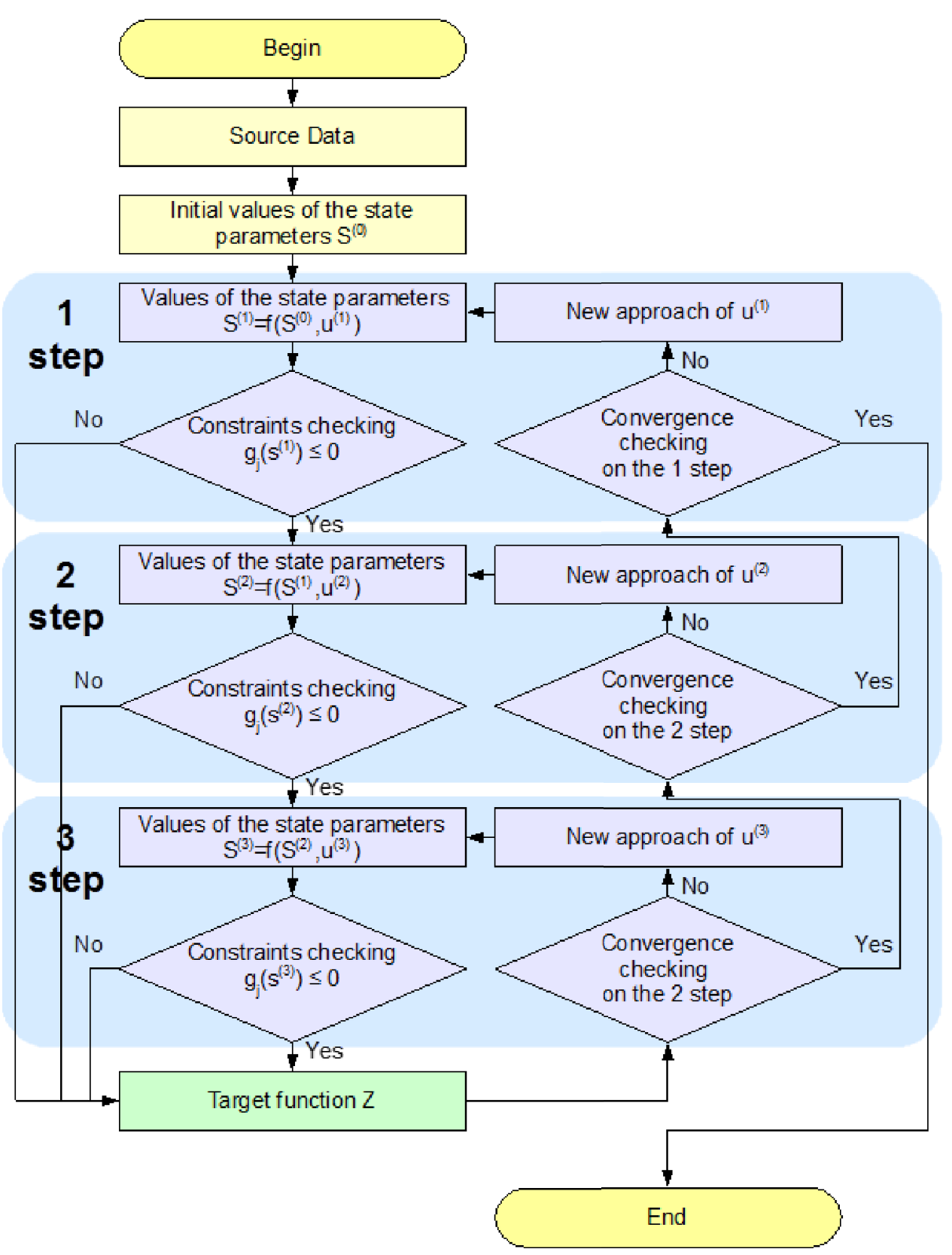

Figure 2. Block diagram of the algorithm for optimizing control of a three-step process. 


$$
F_{j}^{\text {norm }}(u) \text { - the normalized value of the efficiency }
$$
criterion, which is the relative deviation of the current value of this criterion $F_{j}(u)$ (fuel consumption per ton of payload per $1 \mathrm{~km}$ of flight range, traffic handling cost, etc.) from its locally optimal value $F_{j}^{\text {opt }}$ (result of onecriterion optimization). The normalized value of the efficiency criterion is calculated using the following formulas: (criterion minimization - (7), maximization $(8))$ :

$$
\begin{gathered}
F_{j}^{\mathrm{norm}}(u)=\frac{F_{j}(u)-F_{j}^{\mathrm{opt}}}{F_{j}^{\mathrm{opt}}}, \\
F_{j}^{\mathrm{norm}}(u)=\frac{F_{j}^{\mathrm{opt}}-F_{j}(u)}{F_{j}^{\mathrm{opt}}},
\end{gathered}
$$

A set of limitations should be taken into the account during the optimization of the gas turbine engine control, including maximum and minimum airspeed limitations, airframe angle of attack, rotor speeds, turbine inlet temperature limitations and so on.

It also should be noted that some aircraft flight modes are possible only at the corresponding engine modes (e.g. a horizontal flight of an aircraft with a constant speed and maximum commercial load is impossible for the minimal engine operation mode).

As it is not always possible to calculate a flight path to the end and determine the value of the objective function, it is impossible to use standard methods of penalty and barrier functions during conditional optimization of control function. In case of restrictions violation, it is necessary to correct the value of the objective function in such a way that movement towards the violation of restrictions would be unprofitable.

Thus, if the violation of the restrictions (set as $g_{j}\left(S^{(n-1)}, u^{(n)}\right) \leq 0$ ) occured for a current variant of the control function at the i-th step of n-step process, and further calculations are impossible, the calculation of the objective function is performed using the following formula: 9, 10, 12-14, 17, 19-21, 23, 26

$$
Y=10+\frac{n-1}{n}+g_{j}\left(S^{(i-1)}, u^{(i)}\right) .
$$

\section{Conclusion}

Method of optimization of gas turbine engine control function on the basis of dynamic programming is proposed. The continuous flight process is divided into the set of discrete segments and the optimization process is composed of nested parametric conditional optimizations of engine control parameters at the corresponding steps.

The developed method is applicable to various thermodynamic cycles of engine, including complex and variable cycles, transient modes of operation of aircraft and propulsion unit and provides means to define the optimal control program on the basis of flight envelope modelling.

\section{Acknowledgment}

This work was supported by the Ministry of education and science of the Russian Federation in the framework of the implementation of the Program of increasing the competitiveness of Samara University among the world's leading scientific and educational centers for 2013-2020 years.

\section{References}

1. Zhang, X International Conference on Control, Automation and Systems, 556-561. (2017)

2. Singh, V., Sharma, S.K. European Transport Research Review, 7 (2), (2015)

3. Sun, F., Miao, L., Zhang, H. International Journal of Turbo and Jet Engines, (2015)

4. Li, J., Fan, D., Sreeram, V. International Journal of Turbo and Jet Engines, 30 (4), 383-391. (2013)

5. Silva, V.V.R., Fleming, P.J., Sugimoto, J., Yokoyama, R. Applied Soft Computing Journal, 8 (1), 392-401. (2008)

6. Silva, V.V.R., Khatib, W., Fleming, P.J. Engineering Applications of Artificial Intelligence, 18 (5), 575583. (2005)

7. Pérez-Grande, I., Leo, T.J. Applied Thermal Engineering, 22 (17), 1885-1904. (2002)

8. Wang, Y., Xing, Y., Yu, X., Zhang, S. Proceedings of the Institution of Mechanical Engineers, Part G: Journal of Aerospace Engineering, 232 (5), 973-987, (2018)

9. Zheng, Q., Zhang, H. Proceedings of the Institution of Mechanical Engineers, Part G: Journal of Aerospace Engineering, 232 (2), 308-316. (2018)

10. Du, X., Sun, X.-M., Wang, Z.-M., Dai, A.-N. IEEE Access, 5, № 8089348, 24533-24541. (2017)

11. Yu, B., Cao, C., Shu, W., Hu, Z. A IEEE Access, 5, № 8070119, 23848-23857. (2017)

12. Gao, Y., Wang, J., Wu, X., Ye, Z., Hu, Z., Dimirovski, G. Lecture Notes in Electrical Engineering, 359, 205212. (2016)

13. Wang, J., Min, N., Ye, Z., Hu, Z. Chinese Control Conference, CCC, № 6640375, 5368-5372. (2013)

14. Wang, J., Ye, Z., Hu, Z. Journal of Engineering for Gas Turbines and Power, 134 (9), № 094502. (2012)

15. Richter, H., Singaraju, A., Litt, J.S. Journal of Guidance, Control, and Dynamics, 31 (2), 273-281. (2008)

16. Lyantsev, O.D., Breikin, T.V., Kulikov, G.G., Arkov, V.Y. Automatica, 39 (12), 2115-2121. (2003)

17. Frederick, D.K., Garg, S., Adibhatla, S. IEEE Transactions on Control Systems Technology, 8 (6), 961-970. (2000)

18. Xie, Z., Su, M., Weng, S. Applied Thermal Engineering, 21 (1), 111-118. (2001) 
19. V.S. Kuzmichev, A.Yu. Tkachenko, V.N. Rybakov, Proceedings of the Samara Research Center of the Russian Academy of Sciences, 14, 2 (2), 491-494, (2012)

20. E.A. Andreeva, V.M. Tsiruleva, Higher education publishing house, 2006.

21. P. Ahmadi, I. Dincer, M.A. Rosen, Energy Conversion and Management, 76, 282-300, (2013)

22. A. Bensoussan, J. Frehse, S.C.P. Yam, Journal des Mathematiques Pures et Appliquees, 103, 6, 14411474, (2015)

23. H. Zhang, J. Zhang, G.-H. Yang, Y. Luo, IEEE Transactions on Fuzzy Systems, 23, 1, 152-163, (2015)
24. A.J. Chipperfield, B. Bica, P.J. Fleming, IEEE Transactions on Industrial Electronics, 49, 3, 536548, (2002)

25. V.S. Kuz'michev, A.Y. Tkachenko, E.P. Filinov, Y.A. Ostapyuk, I.N. Krupenich, ICMSC 2017, 136-140, (2017)

26. V.S. Kuz'michev, A.Y. Tkachenko, E.P. Filinov, Y.A. Ostapyuk, I.N. Krupenich, V. N. Rybakov, 53rd AIAA/SAE/ASEE Joint Propulsion Conference, №195549, (2017) 